
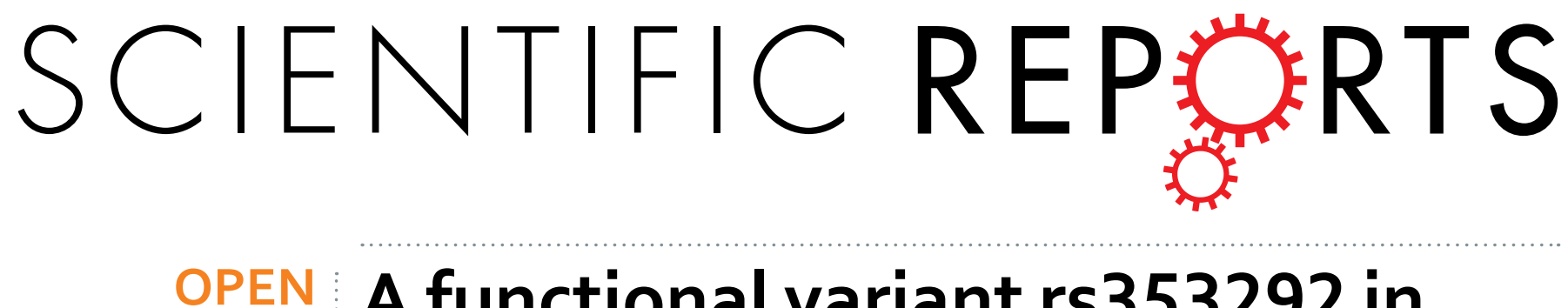

\title{
A functional variant rs353292 in the flanking region of miR-143/145 contributes to the risk of colorectal
}

Received: 14 April 2016

Accepted: 29 June 2016

Published: 22 July 2016

\section{cancer}

\author{
Fang Yuan ${ }^{1, *}$, Ruifen Sun ${ }^{1,2, *}$, Lijuan $\mathrm{Li}^{3}$, Bo Jin ${ }^{3}$, Yanyun Wang ${ }^{4}$, Yundan Liang ${ }^{4}$, Guanglu Che ${ }^{1}$, \\ Linbo $\mathrm{Gao}^{4}$ \& Lin Zhang ${ }^{1,3,4}$
}

MicroRNA (miR)-143 and miR-145 have been identified as molecular regulators in cell proliferation, cell growth, clone formation, apoptosis, cell cycle, invasion, and migration. We previously found that rs353292 in the flanking region of miR-143/145 showed a high frequency in patients with colorectal cancer (CRC). To identify whether the rs 353292 polymorphism is a risk factor for CRC, we conducted this study with larger samples. A total of 809 patients with CRC and 1005 gender matched controls were collected. The rs353292 polymorphism was genotyped by using TaqMan allelic discrimination. Dual luciferase reporter assay was carried out to measure the transcriptional activity. We found that the rs 353292 polymorphism was associated with an increased risk for developing CRC in heterozygous comparison (adjusted $\mathrm{OR}=1.70,95 \% \mathrm{Cl}, 1.32-2.20, P<0.001$ ), dominant genetic model (adjusted $\mathrm{OR}=1.62,95 \% \mathrm{Cl}, 1.26-2.09, P<0.001$ ), and allele comparison (adjusted $\mathrm{OR}=1.46,95 \% \mathrm{Cl}, 1.16-1.84$, $P=0.001)$. The rs $353292 \mathrm{CT} / \mathrm{TT}$ carriers exhibited a lower expression of miR-143 compared to the CC carriers $(P=0.04)$. Moreover, the pGL3-rs353292T displayed a significantly lower luciferase activity than pGL3-rs353292C $(P<0.01)$. These findings indicate that the rs353292 polymorphism is functional and may be a risk factor for the development of CRC.

Colorectal cancer (CRC) is the third most common cancer and the fourth leading cause of cancer death around the world, with an estimated 1.2 million new cases and 600000 deaths per year ${ }^{1,2}$. The highest incidence of CRC is in developed countries of Europe, North America, and Oceania ${ }^{1}$. Recent reports revealed that the incidence is rapidly increasing in many Asian countries including $\mathrm{China}^{3}$. Even with the improvements in diagnosis, surgery, chemotherapy, and targeted therapy, the 5-year survival rate for CRC patients is estimated to be $62-64 \%{ }^{4}$. Previous identified risk factors for CRC included obesity, smoking, dietary patterns, physical inactivity, and genetic factors $^{5-7}$.

MicroRNAs (miRNAs) are a class of conservative endogenous non-coding RNA molecules, with 18-25 nucleotides in length, and have been found in variety of eukaryotic organisms $s^{8}$. Generally, miRNAs play a crucial role in cellular development, differentiation, proliferation, and apoptosis by binding to $3^{\prime}$ untranslated region (UTR) of target mRNA molecules, resulting in the degradation of mRNA and inhibition of protein translation. A series of studies have demonstrated that miRNAs act as potential "tumor suppressors" or oncogenes ${ }^{2,9}$. MiR-143 and miR-145, closely located on 5q33, are co-transcribed from a single promoter, resulting in a primary transcript containing both miRNAs ${ }^{3}$. In 2003, Michael et al. initially reported that the expression of both miRNAs were downregulated in colorectal neoplasia ${ }^{10}$. The two miRNAs are always involved in various cancer-related events

${ }^{1}$ Department of Immunology, West China School of Preclinical and Forensic Medicine, Sichuan University, Chengdu, Sichuan 610041, P.R. China. ${ }^{2}$ Central Laboratory, Yunnan University of Chinese Traditional Medicine, Kunming 650500 , Yunnan, P.R. China. ${ }^{3}$ Department of Forensic Biology, West China School of Preclinical and Forensic Medicine, Sichuan University, Chengdu, Sichuan 610041, P.R. China. ${ }^{4}$ Laboratory of Molecular and Translational Medicine, West China Institute of Women and Children's Health; Key Laboratory of Obstetric \& Gynecologic and Pediatric Diseases and Birth Defects of Ministry of Education; West China Second University Hospital, Sichuan University, Chengdu, Sichuan 610041, P.R. China. *These authors contributed equally to this work. Correspondence and requests for materials should be addressed to L.G. (email: gaolinboscu@163.com) or L.Z. (email: zhanglin@ scu.edu.cn) 


\begin{tabular}{|l|c|c|}
\hline Variables & Cases $\mathbf{n}=\mathbf{8 0 9}(\%)$ & Controls n $=\mathbf{1 0 0 5}(\%)$ \\
\hline Mean age (year) & $61.0 \pm 12.7$ & $48.3 \pm 15.0$ \\
\hline Gender & $482(59.6)$ & $560(55.7)$ \\
\hline Male & $327(40.4)$ & $445(44.3)$ \\
\hline Female & $244(30.2)$ & \\
\hline Tumor site & $563(69.6)$ & \\
\hline Colon & $2(0.2)$ & \\
\hline Rectal & $419(51.8)$ & \\
\hline Both & $390(48.2)$ & \\
\hline Differentiated status & $479(59.2)$ & \\
\hline Well-moderately & $330(40.8)$ & \\
\hline Poorly-undifferentiated & \\
\hline Clinical stage & \\
\hline I-II & $518(64.1)$ & \\
\hline III-IV & \\
\hline Lymph node metastasis & \\
\hline Yes & \\
\hline No &
\end{tabular}

Table 1. Demographics of the CRC Patients and Controls.

\begin{tabular}{|c|c|c|c|c|c|c|}
\hline \multirow[b]{2}{*}{ Genotypes } & \multirow[b]{2}{*}{ Cases n $=809(\%)$} & \multirow[b]{2}{*}{ Controls n $=1005(\%)$} & \multicolumn{2}{|c|}{ Logistic regression (crude) } & \multicolumn{2}{|c|}{ Logistic regression (adjusted $^{\mathrm{a}}$ ) } \\
\hline & & & OR $(95 \% \mathrm{CI})$ & $P$ value & OR $(95 \% \mathrm{CI})$ & $P$ value \\
\hline $\mathrm{CC}$ & $601(74.3)$ & $841(83.7)$ & 1.00 & & 1.00 & \\
\hline CT & $201(24.8)$ & $152(15.1)$ & $\begin{array}{c}1.84 \\
(1.46-2.33)\end{array}$ & $<0.0001$ & $1.70(1.32-2.20)$ & $<0.0001$ \\
\hline $\mathrm{CT} / \mathrm{TT}$ & $208(25.7)$ & $164(16.3)$ & $\begin{array}{c}1.77 \\
(1.41-2.23)\end{array}$ & $<0.0001$ & $1.62(1.26-2.09)$ & $<0.0001$ \\
\hline \multicolumn{7}{|l|}{ Allele } \\
\hline $\mathrm{C}$ & $1403(86.7)$ & $1834(91.2)$ & 1.00 & & 1.00 & \\
\hline $\mathrm{T}$ & $215(13.3)$ & $176(8.8)$ & $\begin{array}{c}1.60 \\
(1.29-1.97)\end{array}$ & $<0.0001$ & $1.46(1.16-1.84)$ & 0.001 \\
\hline
\end{tabular}

Table 2. Association Between the rs353292 Polymorphism and Risk of CRC. adjusted for age and gender using the logistic regression model. OR, odds ratio; CI, confidence interval.

including proliferation, invasion and migration, suggesting a potential possess of anti-tumorigenic activity. With regard to the regulation of miR-143/145, previous reports have shown that KRAS, Ras responsive element binding protein 1, serum response factor, and myocardin can regulate the expression of miR-143/145 by altering the transcription of target genes ${ }^{11-13}$.

Single nucleotide polymorphisms (SNPs), the most common type of genetic variation in human genome, was estimated to disrupt the process of miRNAs, and thus influence their expression. Recently, we discovered several SNPs in the flanking region of miR-143 and miR-145 cluster. Among them, rs353292, located in $673 \mathrm{bp}$ upstream from the start site, was related to an increased risk of $\mathrm{CRC}^{14}$. Since the sample sizes are limited in our previous study ${ }^{14}$, in this study, we replicated the relationship between the rs353292 polymorphism and CRC risk by enlarging sample size. Moreover, dual luciferase reporter assay was used to examine whether the rs353292 polymorphism can affect the transcriptional activity.

\section{Results}

Association of the miR-143/145 rs353292 with risk of CRC. Demographic and clinical characteristics for all the subjects are listed in Table 1. The mean age $( \pm S D)$ of the cases was $61.0 \pm(12.7)$ years, which was higher than the controls $(P<0.001)$. There was no significant difference in gender distribution between cases and controls $(P=0.10)$.

The genotype distributions in the control group followed the HWE $(P=0.09)$. As shown in Table 2 , a significant difference in genotype and allele distributions of the rs353292 was found between cases and controls. A significantly increased CRC risk was found to be associated with CT genotype compared to CC genotype (adjusted $\mathrm{OR}=1.70,95 \% \mathrm{CI}, 1.32-2.20, P<0.001$ ). The CT/TT genotype carriers had a 1.62 -fold increased risk of CRC compared to CC genotype carriers (adjusted OR $=1.62,95 \% \mathrm{CI}, 1.26-2.09, P<0.001$ ). Additionally, the rs353292T allele was associated with a significantly increased risk of CRC compared to C allele (adjusted $\mathrm{OR}=1.46,95 \% \mathrm{CI}, 1.16-1.84, P=0.001)$. Stratification analyses were done according to different variables, including differentiated status, clinical stage, and lymph node metastasis. Nevertheless, no significant association was detected between the rs353292 polymorphism and clinical features (Table 3). 


\begin{tabular}{|l|c|c|c|c|}
\hline Clinical Features & \multicolumn{2}{|c|}{ Genotype Frequency (\%) } & OR (95\% CI) & P value \\
\hline Differentiated status & Well-moderately & Poorly-undifferentiated & & \\
\hline CC & $318(75.9)$ & $283(72.6)$ & 1 & \\
\hline CT/TT & $101(24.1)$ & $107(27.4)$ & $0.84(0.61-1.15)$ & 0.28 \\
\hline C & $734(87.6)$ & $669(85.8)$ & 1 & \\
\hline T & $104(12.4)$ & $111(14.2)$ & $0.85(0.64-1.14)$ & 0.28 \\
\hline Clinical stage & I-II & III-IV & & \\
\hline CC & $357(74.5)$ & $244(73.9)$ & 1 & \\
\hline CT/TT & $122(25.5)$ & $86(26.1)$ & $0.97(0.71-1.34)$ & 0.85 \\
\hline C & $834(87.1)$ & $569(86.2)$ & 1 & \\
\hline T & $124(12.9)$ & $91(13.8)$ & $0.93(0.70-1.24)$ & 0.62 \\
\hline Lymph node metastasis & Yes & No & & \\
\hline CC & $213(73.2)$ & $388(74.9)$ & 1 & \\
\hline CT/TT & $78(26.8)$ & $130(25.1)$ & $1.09(0.79-1.51)$ & 0.59 \\
\hline C & $499(85.7)$ & $904(87.3)$ & 1 & \\
\hline T & $83(14.3)$ & $132(12.7)$ & $1.14(0.85-1.53)$ & 0.39 \\
\hline
\end{tabular}

Table 3. Association Between the rs353292 Polymorphism and Clinical Features of CRC Patients. OR, odds ratio; CI, confidence interval.

Association of the rs 353292 with the expression of miR-143/145. We detected the expression of miR-143 and miR-145 in both CRC tumors and noncancerous tissues. As shown in Fig. 1A,B, levels of both miR-143 and miR-145 in CRC tissues were dramatically lower than those in noncancerous tissues $(P<0.001)$. Furthermore, we analyzed the association between the rs353292 polymorphism and the expression of miR-143 and miR-145 in CRC tissues. We found that the expression of miR-143 in rs353292 CT/TT carriers was significantly lower than that in CC carriers $(P=0.04)$ (Fig. 1C). However, no significant correlation between the rs353292 polymorphism and the expression of miR-145 was found (Fig. 1D).

Dual Luciferase Reporter Assay of the miR-143/145 rs353292 polymorphism. After transient transfection of miR-143/145 flanking region into HCT116, HeLa, and A549 cells, the transcriptional activity was a significantly higher compared to empty plasmid $(P<0.01)$ (Fig. 2A). To further investigate the effect of the rs353292 polymorphism on the transcriptional activity, we constructed two plasmids: pGL3-rs353292T and pGL3-rs353292C. As shown in Fig. 2B, the pGL3-rs353292T showed a significantly lower luciferase activity than pGL3-rs353292C $(P<0.01)$. In HCT116 cells, the rs353292T displayed a 0.45 -fold decreased activity compared to the rs353292C $(P=0.009)$. Similarly reduced luciferase activity was also observed in HeLa and A549 cells, with a fold change of 0.57 and $0.17(P=0.004$ and $P<0.001$, respectively).

\section{Discussion}

We performed a case-control study evaluating the relationship between the rs353292 polymorphism in the flanking region of miR-143/145 and CRC risk. We found that the rs353292 polymorphism was associated with an increased risk for developing CRC in heterozygous comparison, dominant genetic model, and allele comparison. The rs353292 CT/TT carriers exhibited a lower expression of miR-143 compared to the CC carriers $(P=0.04)$. In vitro analysis showed that rs $353292 \mathrm{~T}$ exhibited a lower transcriptional activity compared to rs353292C. These findings suggest that the rs 353292 is functional and may be used as a biomarker for the risk of CRC.

MiR-143 and miR-145 are located on chromosome 5q33.1 in human genome and are speculated to be cotranscribed as the same bicistronic unit ${ }^{15}$. These two miRNAs have been studied extensively and have been identified as "tumor suppressors" in varieties of cancer types, including CRC $C^{8,16-28}$. Downregulation of these miRNAs has been reported in both CRC tissues and CRC cell lines s $^{16-18,20-25,28}$. Such abnormal expression can regulate cell proliferation, cell growth, clone formation, apoptosis, cell cycle, invasion, and migration by targeting lots of genes such as metastasis-associated in colon cancer-1, insulin-like growth factor 1 receptor, DNA methyltranferase 3A, paxillin, toll-like receptor 2, fascin-1, and $K R A S^{16-18,20,21,23-25}$. Furthermore, miR-143 can reduce viability and increase the sensitivity of $\mathrm{CRC}$ cells to 5 -fluorouracil via extracellular-regulated protein kinase $5 /$ nuclear factor- $\kappa \mathrm{B}$ signaling pathway ${ }^{26}$. These findings indicate that miR-143 and miR-145 may be used as not only anti-oncogenic genes but also therapeutic targets for CRC.

Recently, several SNPs in the flanking region of miRNAs have been reported to alter individual's susceptibility to cancer. Among them, an SNP rs4938723 in the promoter of pri-miR-34b/c was the mostly studied, which was reported to be related to the risk of a series of cancer types, such as colorectal cancer ${ }^{29,30}$, hepatocellular carcinoma $^{31}$, gastric cancer ${ }^{32}$, renal cell cancer ${ }^{33}$, esophageal squamous cell carcinoma ${ }^{34}$, nasopharyngeal carcinoma ${ }^{35}$, papillary thyroid carcinoma ${ }^{36}$, and cervical cancer ${ }^{37}$. Other SNPs included rs 10877887 in the promoter of let-7, rs2296616 in the promoter of miR-107, rs999885 in the promoter of miR-106b-25 cluster, and rs4705342 and rs 4705343 in the flanking region of miR-143/145, which were reported to be associated with the risk of papillary thyroid carcinoma ${ }^{38}$, lung cancer ${ }^{39}$, gastric adenocarcinoma ${ }^{40}$, hepatocellular carcinoma ${ }^{41}$, prostate cancer ${ }^{42}$, and cervical squamous cell carcinoma ${ }^{43}$. In our previous study, we reported a new SNP rs353292 in the flanking region of miR-143/145 and the rs353292T allele was a risk factor for the development of $\mathrm{CRC}^{14}$. In this replication study, 

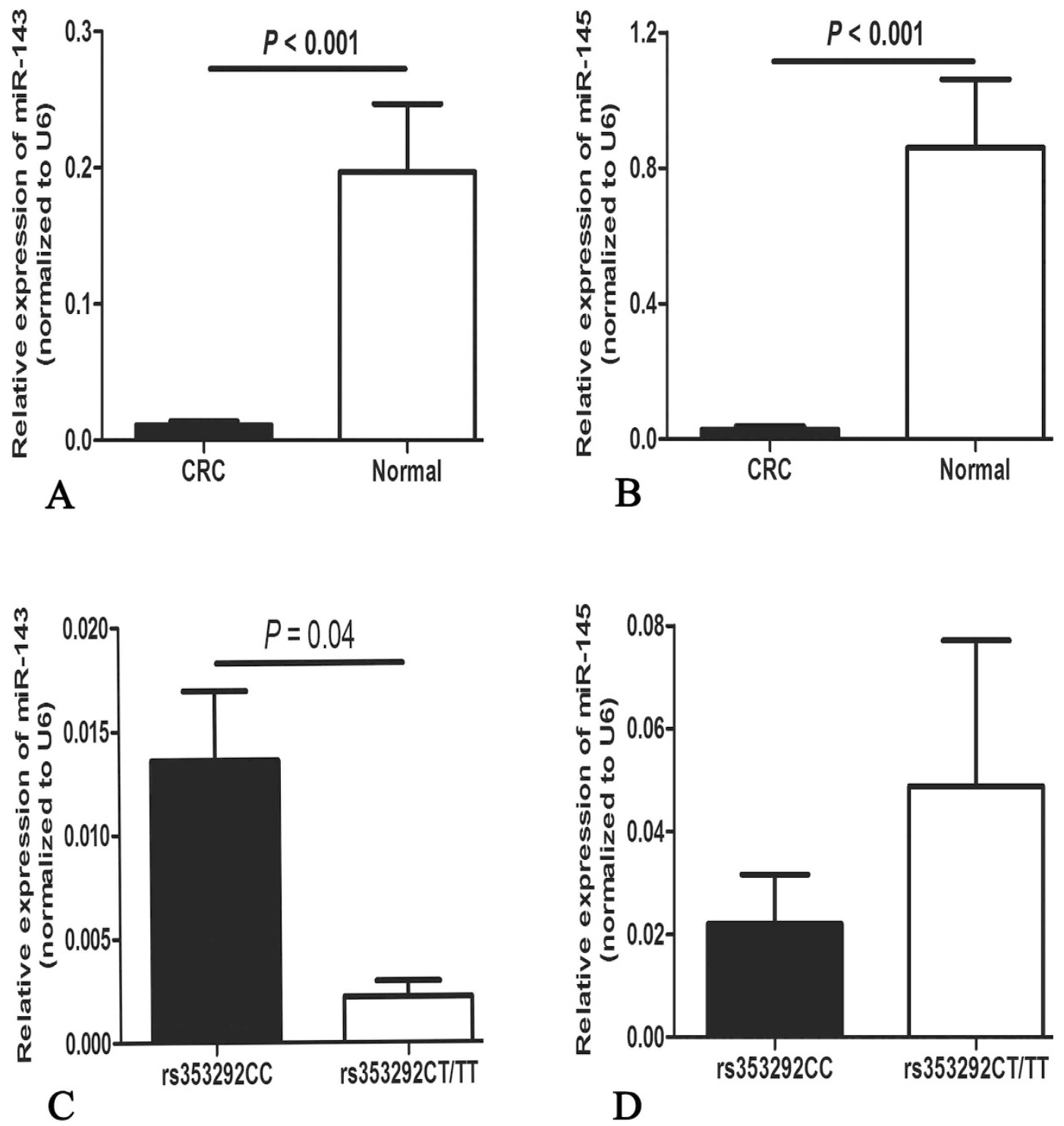

Figure 1. Relative expression of miR-143/145 in 71 CRC tumor tissues and normal tissues determined by qRT-PCR. Both miR-143 (A) and miR-145 (B) were found dramatically lower in CRC tissues $(P<0.001)$. MiR143 was found significantly down-regulated in rs353292 CT/TT carriers (C). No significant relationship of miR145 and the rs353292 polymorphism was found (D).

the same positive effect was also observed. In agreement with the association study, functional analysis revealed that the rs353292T allele had a reduced transcriptional activity in vitro. These findings indicate that the rs $353292 \mathrm{~T}$ allele may result in lower transcriptional activity and reduced level of miR-143, and eventually increase the risk of CRC.

Since DNA for the case group was extracted from paraffin-embedded specimens and DNA for the control group was isolated from peripheral blood samples, we cannot exclude the possibility of heterozygote between cases and controls. And thus confirmation studies should be performed using peripheral blood samples from both case and controls. Moreover, the mean age in the controls was lower than that in cases, which may result in unreliable results due to selection bias. After adjustment by age and gender, however, the positive results were still observed, suggesting the results may be reliable. Well-designed studies are of great importance to verify these findings, especially in other ethnic groups.

In conclusion, we provided the first evidence that the rs353292 in the flanking region of miR-143/145 may modulate transcriptional activity, and finally affect individual's susceptibility to CRC. This work may help us achieve a deeper insight into SNPs in the flanking region of miRNAs relevant to the carcinogenesis of CRC. In the future, in vivo experiments using transgenic mice will be valuable to analyze the rs 353492 polymorphism in the risk of CRC. 


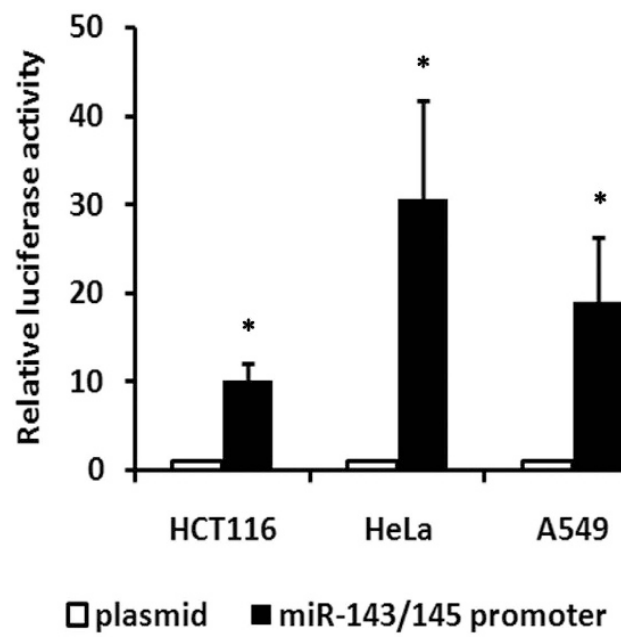

A

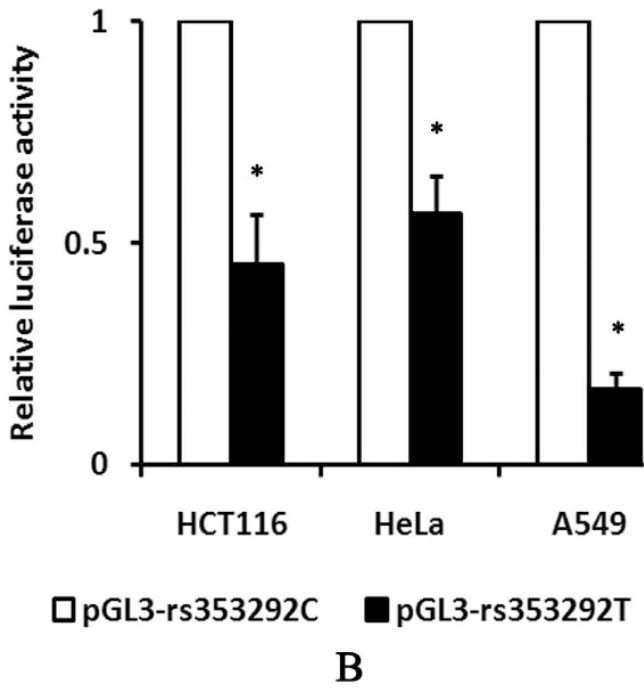

Figure 2. The relative luciferase activity in different cell lines. (A) After transfected with the plasmid containing the flanking region of miR-143/145, significantly higher luciferase activity was observed. (B) The pGL3-rs353292T led to a decrease in luciferase expression compared to the pGL3-rs353292C. Each experiment was done in triplicate and data are presented as mean $\pm \mathrm{SD}$. $* P<0.05$.

\section{Materials and Methods}

Ethics statement. The study protocol was approved by the Ethics Committee of the West China Second University Hospital (Ethics number: 028), and informed consent was obtained from all subjects. All the experiments were performed in accordance with the approved guidelines.

Study population. A total of 809 paraffin wax-embedded CRC samples were consecutively recruited between January 2012 and October 2014 from the Affiliated Hospital of North Sichuan Medical College. Seventy-one fresh CRC tumor tissues and adjacent non-tumor tissues were collected from the Luoyang Central Hospital Affiliated to Zhengzhou University. All patients were diagnosed by histopathological confirmation. Clinical information was collected on age, gender, ethnicity, differentiated status, tumor size, lymph node metastasis, and distant metastasis, through a review of pathological and/or surgical reports. Clinical stage was estimated based on TNM classification. Patients with an evidence of personal or family history of inflammatory diseases or cancer in the intestine were excluded. The control group included 1005 healthy subjects from the hospital for a routine checkup at the same period. All the controls were gender and ethnicity matched to cases.

Genotyping. For the case group, genomic DNA was extracted from paraffin wax-embedded sections; for the control group, genomic DNA was extracted from EDTA- anticoagulated peripheral blood, using commercial extraction kits (Qiagen, Hilden, Germany; Biobeke, Beijing, China). The rs353292 polymorphism was analyzed by using TaqMan allelic discrimination. The SNP assay was validated by sequencing about $5 \%$ of all samples, and the results were $100 \%$ concordant.

RNA extraction and quantitative reverse transcription-PCR assay. Total RNA of tissue samples was extracted using TRIzol reagent according to the manufacturer's instructions (Roche, Indianapolis, IN). $1 \mu \mathrm{g}$ of total RNA was reverse-transcribed to cDNA according to the manufacturer's manual of a commercial kit (Ribobio, Guangzhou, China). MiR-143/145 primers were purchased from Ribobio company (Guangzhou, China). Quantitative real-time PCR was performed using a SYBR green Master Mix (Qiagen, Hilden, Germany). The reaction conditions were as follows: $95^{\circ} \mathrm{C}$ for $10 \mathrm{~min}, 95^{\circ} \mathrm{C}$ for $10 \mathrm{sec}, 60^{\circ} \mathrm{C}$ for $20 \mathrm{sec}, 70^{\circ} \mathrm{C}$ for $10 \mathrm{sec}$ (40 cycles). All reactions were performed in triplicate. Data were normalized using comparative threshold cycle method and U6 for normalization.

Construction of reporter plasmid. Detailed information for constructing reporter plasmids was described in our previous study ${ }^{43}$. Briefly, the fragment containing rs353292T was synthesized by PCR with primers $5^{\prime}$-AGTGGTACCGCCGTGGAGAGTGGAATAGA-3' (forward) and $5^{\prime}$-GTGAAGCTTCCA ACTGACCAGAGATGCAG- $3^{\prime}$ (reverse). The primers were designed to incorporate KpnI and HindIII restriction sites, and the PCR product was cloned into the pGL3-basic vector (Promega, Madison, WI). The rs353292C allele was obtained by using a QuickChange Site-Directed Mutagenesis Kit (Stratagene, La Jolla, CA), and the mutation primers were as follows: 5'-GAAATATCCAGAAAATATACAGACAGATCTATAGAGATA-3' (forward), and 5'-TATCTCTATAGATCTGTCTGTATATTTTCTGGATATTTC-3' (reverse). All constructs were confirmed by direct sequencing (TsingKe, Chengdu, China). We named the generated reporter vectors of pGL3-rs353292T and pGL3-rs353292C, respectively. 
Transfection and dual luciferase reporter assay. HCT116, HeLa, and A549 cells were obtained from our laboratory. All the cell lines were plated on 24 -well plates at a density of $1 \times 10^{5} \mathrm{cells} /$ well. The cells were transiently transfected for $48 \mathrm{~h}$ with $2 \mu \mathrm{g}$ pGL3-basic vector DNA or pGL3-rs353292T or pGL3-rs353292C using $\mathrm{X}$-treme GENE HP reagent (Roche, Indianapolis, IN). According to the manufacturer's protocol, $40 \mathrm{ng} \mathrm{pRL}$-TK vector DNA (Promega, Madison, WI) was co-transfected as an internal control. The luciferase activities were measured on the Dual-Luciferase Reporter Assay System (Promega). The experiment was performed in triplicates and the results were reported as mean \pm standard deviation (SD).

Statistical analysis. The age distribution between the cases and controls was assessed using Student's $t$ test and the gender distribution was compared using chi-square test. Hardy-Weinberg equilibrium (HWE) was tested using chi-square test. The genotype and allele frequencies of the two groups were compared with chi-square test, and the relative risk was estimated by odd ratios (ORs) and 95\% confidence intervals (CIs). The association between the rs 353292 polymorphism and CRC was adjusted by age and gender using logistic regression model. The levels of luciferase reporter gene expression were examined using Student's $t$ test between different constructs. Statistical analyses were performed using the software Statistical Package for Social Science (SPSS) for Windows, version 19.0 (SPSS, Inc., Chicago, IL). A $P$ value $<0.05$ was considered statistically significant.

\section{References}

1. Brenner, H., Kloor, M. \& Pox, C. P. Colorectal cancer. Lancet 383, 1490-1502, doi: 10.1016/S0140-6736(13)61649-9 (2014)

2. Farazi, T. A., Spitzer, J. I., Morozov, P. \& Tuschl, T. miRNAs in human cancer. The Journal of pathology 223, 102-115, doi: 10.1002/ path.2806 (2011).

3. Kent, O. A., Fox-Talbot, K. \& Halushka, M. K. RREB1 repressed miR-143/145 modulates KRAS signaling through downregulation of multiple targets. Oncogene 32, 2576-2585, doi: 10.1038/onc.2012.266 (2013).

4. Siegel, R., Ward, E., Brawley, O. \& Jemal, A. Cancer statistics, 2011: the impact of eliminating socioeconomic and racial disparities on premature cancer deaths. CA: a cancer journal for clinicians 61, 212-236, doi: 10.3322/caac.20121 (2011).

5. Botteri, E. et al. Smoking and colorectal cancer: a meta-analysis. Jama 300, 2765-2778, doi: 10.1001/jama.2008.839 (2008).

6. Lee, I. M. et al. Effect of physical inactivity on major non-communicable diseases worldwide: an analysis of burden of disease and life expectancy. Lancet 380, 219-229, doi: 10.1016/S0140-6736(12)61031-9 (2012).

7. Zoratto, F. et al. Focus on genetic and epigenetic events of colorectal cancer pathogenesis: implications for molecular diagnosis. Tumour biology: the journal of the International Society for Oncodevelopmental Biology and Medicine 35, 6195-6206, doi: 10.1007/ s13277-014-1845-9 (2014).

8. Bartel, D. P. MicroRNAs: genomics, biogenesis, mechanism, and function. Cell 116, 281-297, doi: S0092867404000455 (2004).

9. Melo, S. A. \& Esteller, M. Dysregulation of microRNAs in cancer: playing with fire. FEBS letters 585, 2087-2099, doi: 10.1016/j. febslet.2010.08.009 (2011).

10. Michael, M. Z., SM, O. C., van Holst Pellekaan, N. G., Young, G. P. \& James, R. J. Reduced accumulation of specific microRNAs in colorectal neoplasia. Mol Cancer Res 1, 882-891 (2003).

11. Rangrez, A. Y., Massy, Z. A., Metzinger-Le Meuth, V. \& Metzinger, L. miR-143 and miR-145: molecular keys to switch the phenotype of vascular smooth muscle cells. Circulation. Cardiovascular genetics 4, 197-205, doi: 10.1161/CIRCGENETICS.110.958702 (2011).

12. Xin, M. et al. MicroRNAs miR-143 and miR-145 modulate cytoskeletal dynamics and responsiveness of smooth muscle cells to injury. Genes Dev 23, 2166-2178, doi: gad.1842409 (2009).

13. Kent, O. A. et al. Repression of the miR-143/145 cluster by oncogenic Ras initiates a tumor-promoting feed-forward pathway. Genes Dev 24, 2754-2759, doi: 24/24/2754 (2010).

14. Li, L. et al. Association between polymorphisms in the promoter region of miR-143/145 and risk of colorectal cancer. Hum Immunol 74, 993-997, doi: 10.1016/j.humimm.2013.04.019 S0198-8859 (13)00105-5 (2013).

15. Cordes, K. R. et al. miR-145 and miR-143 regulate smooth muscle cell fate and plasticity. Nature 460, 705-710, doi: 10.1038/ nature08195 (2009).

16. Zhang, Y. et al. MicroRNA-143 targets MACC1 to inhibit cell invasion and migration in colorectal cancer. Molecular cancer 11, 23, doi: 10.1186/1476-4598-11-23 (2012).

17. Su, J. et al. MiR-143 and MiR-145 regulate IGF1R to suppress cell proliferation in colorectal cancer. PLoS One 9, e114420, doi: 10.1371/journal.pone.0114420 (2014).

18. Ng, E. K. et al. MicroRNA-143 targets DNA methyltransferases 3A in colorectal cancer. Br J Cancer 101, 699-706, doi: 6605195 (2009).

19. Kamatani, A. et al. Downregulation of anti-oncomirs miR-143/145 cluster occurs before APC gene aberration in the development of colorectal tumors. Medical molecular morphology 46, 166-171, doi: 10.1007/s00795-013-0020-5 (2013).

20. Qin, J. et al. MicroRNA-145 suppresses cell migration and invasion by targeting paxillin in human colorectal cancer cells. International journal of clinical and experimental pathology 8, 1328-1340 (2015).

21. Yang, F., Xie, Y. Q., Tang, S. Q., Wu, X. B. \& Zhu, H. Y. miR-143 regulates proliferation and apoptosis of colorectal cancer cells and exhibits altered expression in colorectal cancer tissue. International journal of clinical and experimental medicine 8, 15308-15312 (2015).

22. Iio, A., Nakagawa, Y., Hirata, I., Naoe, T. \& Akao, Y. Identification of non-coding RNAs embracing microRNA-143/145 cluster. Mol Cancer 9, 136, doi: 1476-4598-9-136 (2010).

23. Guo, H. et al. The regulation of Toll-like receptor 2 by miR-143 suppresses the invasion and migration of a subset of human colorectal carcinoma cells. Molecular cancer 12, 77, doi: 10.1186/1476-4598-12-77 (2013).

24. Feng, Y. et al. MicroRNA-145 inhibits tumour growth and metastasis in colorectal cancer by targeting fascin-1. British journal of cancer 110, 2300-2309, doi: 10.1038/bjc.2014.122 (2014).

25. Chen, X. et al. Role of miR-143 targeting KRAS in colorectal tumorigenesis. Oncogene 28, 1385-1392, doi: onc2008474 (2009).

26. Borralho, P. M. et al. MicroRNA-143 reduces viability and increases sensitivity to 5-fluorouracil in HCT116 human colorectal cancer cells. FEBS J 276, 6689-6700, doi: EJB7383 (2009).

27. Yuan, W. et al. Up-regulation of microRNA-145 associates with lymph node metastasis in colorectal cancer. PloS one 9, e102017, doi: 10.1371/journal.pone.0102017 (2014).

28. Slaby, O. et al. Altered expression of miR-21, miR-31, miR-143 and miR-145 is related to clinicopathologic features of colorectal cancer. Oncology 72, 397-402, doi: 000113489 (2007).

29. Gao, L. B. et al. A genetic variant in the promoter region of miR-34b/c is associated with a reduced risk of colorectal cancer. Biol Chem 394, 415-420, doi: 10.1515/hsz-2012-0297/j/bchm.just-accepted/hsz-2012-0297/hsz-2012-0297.xml (2013).

30. Oh, J. et al. Polymorphisms of the pri-miR-34b/c promoter and TP53 codon 72 are associated with risk of colorectal cancer. Oncol Rep 31, 995-1002, doi: 10.3892/or.2013.2926 (2014).

31. Xu, Y. et al. A potentially functional polymorphism in the promoter region of miR-34b/c is associated with an increased risk for primary hepatocellular carcinoma. Int J Cancer 128, 412-417, doi: 10.1002/ijc.25342 (2011). 
32. Pan, X. M. et al. Pri-miR-34b/c rs4938723 Polymorphism Is Associated with a Decreased Risk of Gastric Cancer. Genet Test Mol Biomarkers 19, 198-202, doi: 10.1089/gtmb.2014.0287 (2015).

33. Zhang, S. et al. A potentially functional polymorphism in the promoter region of miR-34b/c is associated with renal cell cancer risk in a Chinese population. Mutagenesis 29, 149-154, doi: 10.1093/mutage/geu001 (2014).

34. Yin, J. et al. Hsa-miR-34b/c rs4938723 T > C and hsa-miR-423 rs6505162 C > A polymorphisms are associated with the risk in a Chinese population. PLoS One 8, e80570, doi: 10.1371/journal.pone.0080570 (2013).

35. Li, L. et al. Interactions of miR-34b/c and TP-53 polymorphisms on the risk of nasopharyngeal carcinoma. Tumour Biol 34, 1919-1923, doi: 10.1007/s13277-013-0736-9 (2013).

36. Chen, P. et al. Pri-Mir-34b/C and Tp-53 Polymorphisms are Associated With The Susceptibility of Papillary Thyroid Carcinoma: A Case-Control Study. Medicine 94, e1536, doi: 10.1097/MD.0000000000001536 (2015).

37. Yuan, F. et al. Combined analysis of pri-miR-34b/c rs4938723 and TP53 Arg72Pro with cervical cancer risk. Tumour Biol, doi: 10.1007/s13277-015-4467-y (2015).

38. Wang, Y. et al. Association Between Genetic Polymorphisms in the Promoter Regions of Let-7 and Risk of Papillary Thyroid Carcinoma: A Case-Control Study. Medicine 94, e1879, doi: 10.1097/MD.0000000000001879 (2015).

39. Shen, L. Q. et al. A single nucleotide polymorphism in the promoter region of let-7 family is associated with lung cancer risk in Chinese. Genet Mol Res 14, 4505-4512, doi: 10.4238/2015.May.4.8 (2015).

40. Wang, S. et al. A common genetic variation in the promoter of miR-107 is associated with A common genetic variation in the promoter of miR-107 is associated with gastric adenocarcinoma susceptibility and survivalsusceptibility and survival. Mutat Res 769, 35-41, doi: 10.1016/j.mrfmmm.2014.07.002 (2014).

41. Liu, Y. et al. A genetic variant in the promoter region of miR-106b-25 cluster and risk of HBV infection and hepatocellular carcinoma. PLoS One 7, e32230, doi: 10.1371/journal.pone.0032230 PONE-D-11-14564 (2012).

42. Chu, H. et al. A functional variant in miR-143 promoter contributes to prostate cancer risk. Arch Toxicol 90, 403-414, doi: 10.1007/ s00204-014-1396-2 (2016).

43. Liang, Y. et al. A Functional Polymorphism in the Promoter of MiR-143/145 Is Associated With the Risk of Cervical Squamous Cell Carcinoma in Chinese Women: A Case-Control Study. Medicine 94, e1289, doi: 10.1097/MD.0000000000001289 (2015).

\section{Acknowledgements}

This work was supported by the National Natural Science Foundation of China (no. 81302149), the Ph.D. Programs Foundation of Ministry of Education of China (no. 20130181120011), the Science and Technology Pillar Program of Sichuan Province (no. 2016SZ0013), Distinguished Young Scientist of Sichuan University (no. 2013SCU04A38).

\section{Author Contributions}

F.Y. and R.S. designed and conducted the experiment, L.L., B.J. and Y.W. collected samples, Y.L. and G.C. prepared figures, L.G. and L.Z. wrote and reviewed the manuscript. All authors approved the final version of the submitted paper.

\section{Additional Information}

Competing financial interests: The authors declare no competing financial interests.

How to cite this article: Yuan, F. et al. A functional variant rs353292 in the flanking region of miR-143/145 contributes to the risk of colorectal cancer. Sci. Rep. 6, 30195; doi: 10.1038/srep30195 (2016).

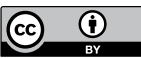

This work is licensed under a Creative Commons Attribution 4.0 International License. The images or other third party material in this article are included in the article's Creative Commons license, unless indicated otherwise in the credit line; if the material is not included under the Creative Commons license, users will need to obtain permission from the license holder to reproduce the material. To view a copy of this license, visit http://creativecommons.org/licenses/by/4.0/

(c) The Author(s) 2016 